\title{
CATALOGUES OF PLANETARY NEBULAE
}

\author{
Agnès Acker \\ Observatoire, Centre de Données de Strasbourg \\ 11, Rue de I'Université \\ 67000 Strasbourg \\ France
}

ABSTRACT. Firstly, the general requirements concerning catalogues are studied for planetary nebulae, in particular concerning the objects to be included in a catalogue of $\mathrm{PN}$, their denominations, followed by reflexions about the afterlife and computerized versions of a catalogue. Then, the basic elements constituting a catalogue of PN are analyzed, and the available data are looked at each time.

\section{A. INTRODUCTION}

The principal aim of a catalogue is to identify a large number of objects having the same nature, and to present and preserve all observational data available for these objects. Planetary nebulae (PN) are particularly interesting to catalogue. The sample of observable $P N$ constitutes a highly representative fraction of the PN system in the Galaxy; as the detection and analysis of PN is done essentially using emission lines, the survey can be done at great distance, enabling the group to be studied as representing a population of the old disk of the Galaxy.

Planetary nebulae are fascinating objects, varied, not easily classifiable, interesting in all spectral regions, each area bringing some specific information (dust in the infrared, molecules in the radio, the central star in the ultraviolet and the visible, ...), from all of which the object called "planetary nebula" can be perceived and described.

As of today, two main catalogues concerning PN exist: "The Catalogue of Galactic Planetary Nebulae" (CGPN), by Perek and Kohoutek (1967), containing 1036 objects and 477 bibliographical references, and "The Catalogue of the Central Stars of True and Possible PN" by Acker et al (1982), containing 480 stars of which 95 have been discovered after 1967, and 894 references. The other existing catalogues give only specific data of samples of PN observed in various spectral ranges. Before listing the catalogues presently available, I shall go over general characteristics defining a catalogue. 


\section{B. DEFINITION AND ELEMENTS OF A CATALOGUE}

1. Jaschek (1984), speaking as head of the "Centre de Données de Strasbourg", proposes a precise terminology for astronomical catalogues, because of the different meanings with which the word "catalogue" is used:

- observational catalogues, or lists of an author's own observations, such as Hipparchus' Catalogue, the first surviving star catalogue; or the Wray study of $\mathrm{H}_{\alpha}$ emission objects (1966);

- bibliographic compilation catalogues, or lists of observations from many different sources, such as the Sabbadin Catalogue of expansion velocities for planetary nebulae (1984);

- critical compilation catalogues, or those providing a critical evaluation of the observations collected, and possible average values, such as the Schneider, Terzian et al catalogue of radial velocities for planetary nebulae (1983).

Jaschek pointed out that all these meanings have several elements in common:

- the purpose of the catalogue must be made known to the reader;

- data presented in a large number must be ordered;

- the data should be clearly specified.

Let us return to these three requirements.

a) The purpose of the catalogue must be clearly known, defining the kind of data and/or objects for which the catalogue is made.

For instance, to what extent should other objects, which are only possible $\mathrm{PN}$, or which can be related to PN for various reasons, be included in a catalogue of PN ? Examples of the first instance are objects which are not well known and whose observational characteristics are common to several types of object: a small object of very low excitation could be a young PN or a compact HII region; an emission-line spectrum showing habitual nebular lines and other peculiar features could be emitted by a peculiar PN or by a Seyfert-Galaxy (unidentifiable if the redshift cannot be calculated), or by a symbiotic star (unidentifiable if the red part of the spectrum remains unknown). This explains how, among the 1036 objects of the CGPN, more than 150 which have been better studied since, have turned out to be other kinds of objects. Similarly, among the 586 objects in the Wray lists (1966) which could be related to PN, there are more than 100 for which no finding chart exists nor any observation other than Wray's, and which could be "not PN". The same uncertainty is found in other lists (for example, possible PN detected only through IRAS colors or radio measurements).

On the other hand, there exist objects presumed to be "protoplanetaries", difficult to identify as the evolutionary process leading to the formation of PN is poorly understood. About 40 stars are called proto PN (such as HBV 475, V1016 Cyg, HM Sge, with a symbiotic behaviour, or objects from IRC and CRL lists); one could add the twenty or so $\mathrm{OH} / \mathrm{IR}$ like sources detected by IRAS. 
In my opinion, a catalogue of PN could present these two types of objects, possibly $\mathrm{PN}$ or related to $\mathrm{PN}$, in the form of lists in annex.

b) The data must be ordered, the commonest ordering being by positional (equatorial or galactic) coordinates. So as to be able to make the link between different catalogues, two identifiers are needed for each object: an "internal" one, fairly arbitrary, but unaffected by positional errors (for example: "Dumbbell nebula", or M 27), and an "external" one, more objective, generally based on the coordinates referring to a given equinoxe $\left(60-3^{\circ} 1\right.$ corresponding to the 1950 galactic longitude and latitude given for the above object).

The record number of denominations is held by $P N 349+101$ which has accumulated 11 names: NGC 6302, ARO 502, ESO 392-05, Gum 60, He 2-204, RCW 124, Sa 2-180, Sh 1-3, Sh 2-6, VV 94, VV' 168, Wray 16-259. The best named central star is the nucleus of $64+5^{\circ} 1=A G+301906.0, B D+303639$, Campbell's star GCRV 11983 ,HD 184738, KY 9296, LS II +30 04,PLX 4591.

As the various catalogues each use their own "internal" ordering, without correlations between them, a cross-identification is necessary, relating one internal identifier to another internal one, and to an unique external identifier. For planetary nebulae, the external identifier is based on galactic coordinates, as proposed by Perek and Kohoutek (1967). For the internal names, abbreviations of the discovery lists should be explained to avoid confusion. Take, for example, the abbreviation "St"; it corresponds to Stephenson, not to Sanduleak, who is indicated as "Sa" (not "Sp" which is the abreviation for Shapley). The object 284- $5^{\circ} 1$, therefore, is given the name Sa 1-1, replacing St 1-1 or Sp 1-1.

The IAU-sponsored "First dictionary of nomenclature of celestial objects", by Fernandez, Lortet and Spite (1983) provides a complete list of abbreviations whose use will resolve most ambiguities; for planetary nebulae, the index of 86 discovery lists and the cross-identification given by Acker et al (1983) for 1518 objects called "PN" are in accordance with it.

c) The data should be properly specified, so as to make it possible to use them again. In particular, the observations must be located in time and space via a "journal of observations", and the description of how the data were obtained should be given (or a bibliographic reference should supply the missing information). For example, a list of spectrophotometric observations of nebulae (intensities relative to $\mathrm{H} \beta$ ) can be useful only if the spectral resolution, as well as the aperture, the location and the orientation of the slit are specified. Besides this, the specification of what errors are quoted (internal ? external ? mean ? ... ?) must also be explained, as must the procedures used to obtain the average data listed in compilation catalogues. The additional information given in the "notes" should be explicit; a remark like "the spectrum is peculiar", without other comment, is practically useless.

2. What is the afterlife of a catalogue ? It is conditioned by the inevitable errors which it contains, and by the new data which appear after its publication. The first "errors" concern the objects themselves; the status of a PN being very difficult to define, especially if few data are available, a lot of objects 
given as "PN" in various lists in fact turn out to be symbiotic stars or galaxies or other kinds of object. Other errors may be typographical or clerical, misidentification (BD-120133 instead of $B D-12^{\circ} 134$ for the nucleus of NGC 246), or wrong data (the visual magnitude of the central star of NGC 2440, accepted as near to 14 up until 1986, is in fact much fainter, near to 19). As noted by Jaschek, once the error is detected, it usually has a long, happy life, because scientific journals do not like to publish errata, and because, even if published, errata are largely ignored by users. Thus, we still find the denominational error attributing to PN $164+31^{\circ} 1=V V 47$ the name NGC 247475 which refers to a nearby pair of galaxies.

As observations are mainly taken with the help of a catalogue, a catalogue becomes out of date all the faster according to how long it has been awaited and how good it is! This explains how, since the publication of Perek and Kohoutek's catalogue, the annual number of publications devoted to planetary nebulae rose from 35 in 1965-67 to 102 in 1968-70 (see fig.1). The large amount of relevant data is more and more difficult to present in its entirety in a catalogue; especially when it is a case of complex images in two dimensions; in this case it is therefore impossible to give in a catalogue more than the title ("label") of a work, and its bibliographical reference.

3. Updating and error correction are facilitated in computerized versions of a printed catalogue, and such versions also give rapid access to stored data and sampling facilities. However, the lifetime of a magnetic tape seems to be about one order of magnitude shorter than that of a printed catalogue, and another disadvantage pointed out by Jaschek is that the computer version escapes the control of its author, being very easily changeable by anyone who has access to the tape; the replacement of tapes by videodisks should overcome some of these difficulties. Even so, it is never possible to give the same information in a computer version as in printed versions : for instance, only printed versions can contain finding charts, spectroscopic recordings, detailed qualitative notes and introductions, ...

Figure 1 -

Evolution of the annual number of papers devoted to planetary nebulae

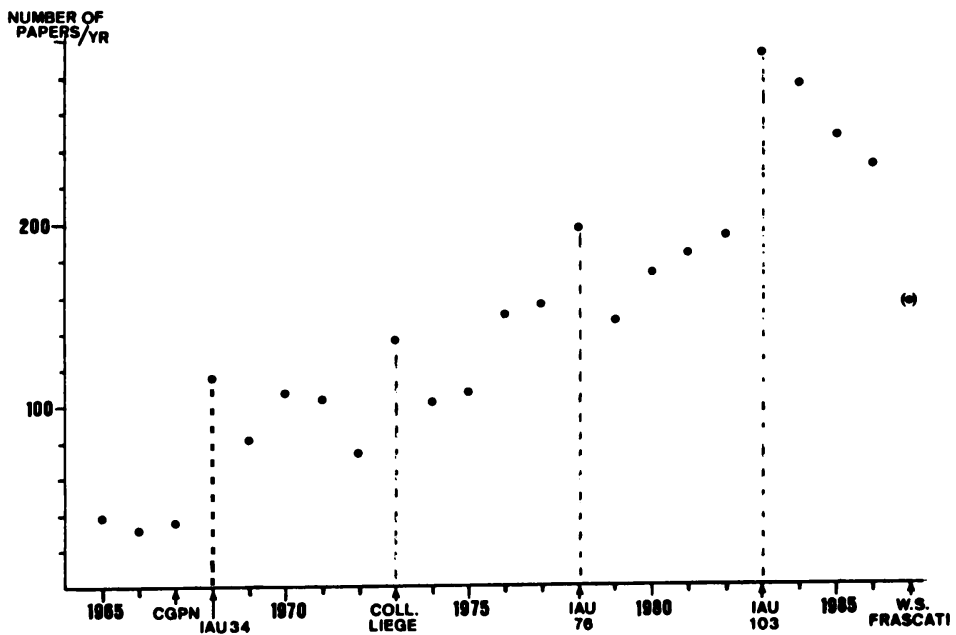




\section{BASIC ELEMENTS OF A CATALOGUE OF PLANETARY NEBULAE}

The essential observational data which should appear in a catalogue of PN concern the position and the structure, velocities, fluxes in various spectral ranges, and the central stars.

\section{Positions and identification: dimensions and morphology}

- Optical coordinates of a large number of PN have been determined since 1969 by various authors (Table 1), by measuring the positions of the PN on Palomar or ESO sky survey prints or plates, the calibration being done using known stars in each field. The accuracy is of about 0.1 arcmn.

Table 1 - OPTICAL COORDIMATES OF PLANETARY nEbULAE

\begin{tabular}{|c|l|}
\hline $\begin{array}{c}\text { Number of } \\
\text { objects }\end{array}$ & \multicolumn{1}{|c|}{ References } \\
\hline 32 & Higgs L.A., 1969, J. Roy. Astron. Soc. Can. 63, 200-202 \\
153 & Milne D.K., 1973, Astron. J. 78, 239-242 \\
345 & Milne D.K., 1976, Astron. J. 81, 753-758 \\
84 & Blackwell S.R., Purton C.R., 1981, Astron. Astrophys. Suppl. 46, 181-183 \\
722 & Lauberts A., 1982, The ESO/Uppsala Survey of the ESO (B) Atlas. \\
\hline
\end{tabular}

The positions are also determined from the radio maps for about 450 objects by the authors cited in Table 4. The VLA position is given at \pm 0.5 arcsec; these coordinates can be quite different from the optical ones, in particular because the maximum emission is not the same in various spectral ranges (in particular, in the case of IC 2120). The same remark can be applied to the coordinates supplied by IRAS (divergence with the optical positions for 4$\left.2^{\circ} 1,254+5^{\circ} 1,299-1^{\circ} 1\right)$.

- The identification of the PN on "finding charts" is unclear or inexistentin 230 cases (16\% of the whole sample), in particular for the crowded area in the Milky Way and for the objects without an identification chart in the discovery papers. This situation will be clarified for most of the objects observed by Acker and Stenholm (spectroscopic survey); besides this, A. GutierrezMoreno, B.M. Lasker and T.D. Kinman have completed 43 sets of photographs in the direction of the galactic center, and are preparing a second program of 52 sets.

New finding charts will be prepared by C. Schohn for the Strasbourg - ESO Catalogue of PN; southern and equatorial PN will be identified on ESO and SRJ plates; but a homogenous solution has not yet been found for the northern PN (about 400), as the new Palomar Sky Survey is not yet ready.

- The dimensions and structure of PN are poorly known, for two main reasons: - the nebular contours are badly defined, as the dimension and the morphology differs from one ion to the other, and therefore depend on the spectral area of the observation and on the exposure time. In addition, radio pictures are often much more symetric than optical ones, because the extinction in the visible is quite strong (case of NGC 7027).

- PN are distant objects; almost half of the known PN are unresolved, starlike 
objects, obviating the study of any properties that require knowledge of their angular sizes; these include the absolute fluxes, the mass, the distance, ...

An effort has been made in this direction, thanks to radio interferometric observations, which have been able to resolve very compact PN and to determine their angular size with an angular resolution of 0.1 to 0.5 arcsec (see Terzian's work since 1979; later: Isaacman, 1984; Kwok, 1981 and 1985 - references in Table 4).

Other substantial progress concerning the dimensions and the morphology of PN has been made in the optical field with the help of CCD images; about $100 \mathrm{PN}$ have been measured up to now, using this technique, in particular by Jewitt, Chu, Grewing and their collaborators. It has been found that about $20 \%$ of the well-studied PN have two or even more shells, and are very often surrounded by large halos.

For each value given for the dimension in a catalogue, it is indispensable to specify whether it concerns a radio or optical measurement. Morphological information can be specified by a summary description (no definitive classification exists up to now); but above all by a high quality image given in the "Finding Charts".

\section{Radial and expansion velocities}

Contrary to the shape and size of $\mathrm{PN}$, velocities are objective data, which can be determined without ambiguity. The radial velocities of $532 \mathrm{PN}$ have been presented in the catalogue of Schneider et al (1983), who have made a critical study of all the values published in the literature and have calculated mean values. New radial velocities have since been published for about fifteen PN.

Expansion velocities have been compiled for 165 PN in the catalogue of Sabbadin (1984), and more recently by Weinberger who has now listed 237 objects in his unpublished catalogue.

\section{Fluxes and line intensities}

These very important data have been increasing over the last ten years, due to more rapid and luminous instruments, and due to the amount of spatial results (IUE, IRAS).

- Absolute $H \beta$ fluxes are known today for about 450 objects (see Table 2); $\mathrm{Kaler}$ and $\mathrm{C}$ ahn have developed an unpublished catalogue of data relative to the distances and temperatures of $P N$, listing in particular absolute $H \beta$ fluxes, with errors, corrected to the modern photometric standard.

- Relative line intensities in the optical range

The reference work is Kaler's catalogue (1976), giving line strengths for 335 PN, as observed by various workers; Kaler is still updating this compilation, and has plans to publish a second edition of his catalogue. Spectroscopic surveys are conducted by several authors, in particular by Aller and Keyes (results 
Table 2 - ABSOLUTE H $\beta$ FLUES

\begin{tabular}{|c|c|}
\hline $\begin{array}{l}\text { Number of } \\
\text { objects }\end{array}$ & References \\
\hline 116 & Perek L., 1971, B.A.C. 22, 103-107 \\
\hline$\approx 300$ & Cahn J., Kaler J.B., 1971, Astrophys. J. Suppl. 22, 319-368 \\
\hline 25 & Kaler J.B., 1976, Astrophys. J., 210, 113-119 \\
\hline 33 & Torres-Peimbert S., Peimbert M., 1977, Rev. Mex. Astron. 2, 181-207 \\
\hline 44 & Barker T., 1978, Astrophys. J., 219, 914-930 \\
\hline 19 & Kaler J.B., 1980; Astrophys. J. 239, 78-88 \\
\hline 30 & Kohoutek L., Martin W., 1981, Astron. Astrophys. Suppl. 44, 325-328 \\
\hline 55 & Carrasco L., Serrano A., Costero R., 1983, Rev. Mex. Astron. 8, 187 \\
\hline 72 & Webster B.L., 1983, P.A.S.P. 95, 610-613 \\
\hline 31 & Kaler J.B., 1983, Astrophys. J. 264, 594-598 \\
\hline 57 & Kaler J.B., 1983, Astrophys. J. 271, 188-220 \\
\hline 49 & Cahn J., 1984, Astrophys. J. 279, 304-309 \\
\hline 51 & Shaw R.A., Kaler J.B., 1985, Astrophys. J., 295, 537-546 \\
\hline 134 & Shaw R.A., 1985, Thesis, University of Illinois at Urbana-Champaign \\
\hline 14 & Gutierrez-Moreno A., Moreno H., Cortes C., 1985, P.A.S.P. 97, 397-403 \\
\hline 13 & $\begin{array}{l}\text { Viadana L., de Freitas Pacheco J.A., 1985, Publ. Observatorio Nacional } \\
\text { CNPq - Brazil, No } 10\end{array}$ \\
\hline 102 & $\begin{array}{l}\text { Acker A., Stenholm B., 1987, Spectroscopic survey of planetary nebulae, } \\
\text { private communication }\end{array}$ \\
\hline For & ts, the absolute $H B$ flux has been measured \\
\hline
\end{tabular}

Table 3 - INFRARED DATA

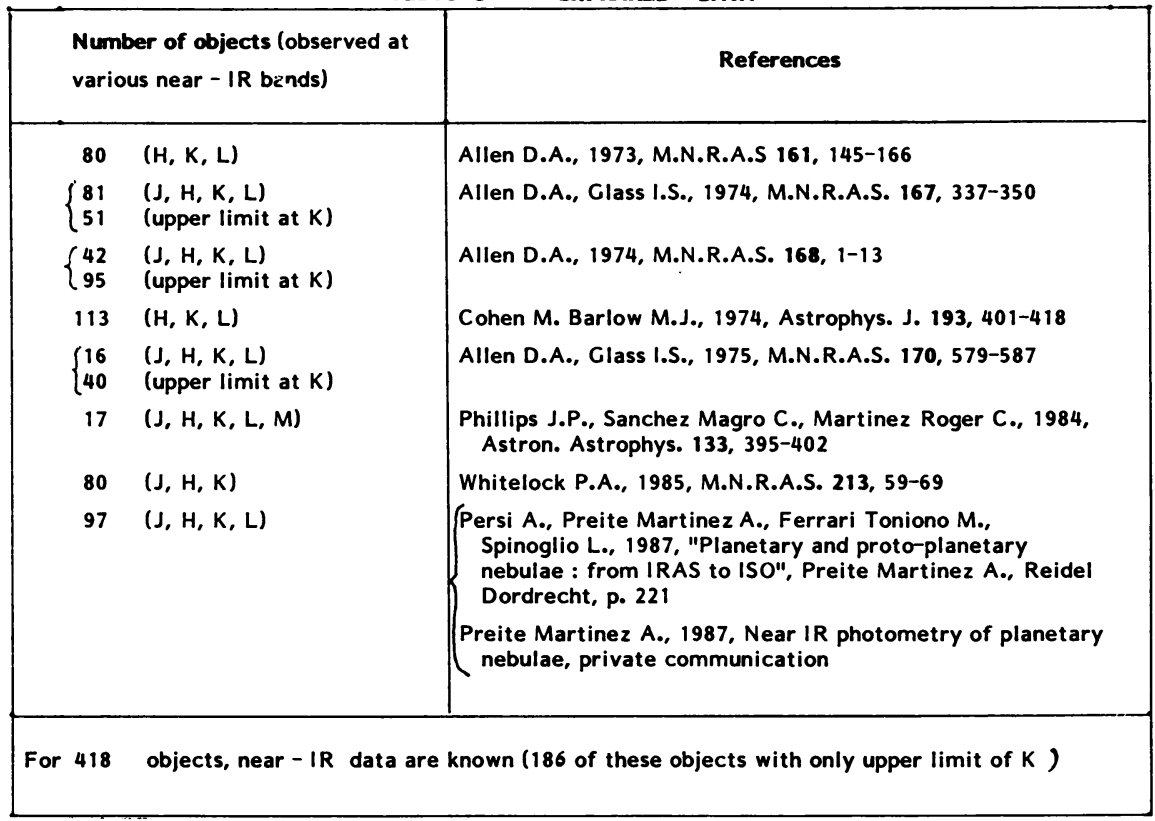


published for 51 PN up to now), and Acker and Stenholm (results so far obtained for 750 PN at La Silla, ESO, Chile, and 180 PN at the Observatoire de HauteProvence, France).

\section{- Ultraviolet fluxes}

In 1985, more than 150 PN have been observed with IUE; results have appeared in various papers, but an "atlas of IUE spectra of PN" has not yet appeared. In the forthcoming PN catalogue, only the IUE Merged Log will be given, specifying high- or low-resolution spectra.

\section{- Infrared fluxes}

As shown on Table 3, more than 400 objects have up to now been observed in the near-IR; but reliable data are known for only about half of these objects. In addition, from 1981 to 1986, several authors (Aitken, Roche, Whitmore) have done $8-13 \mu \mathrm{m}$ spectral observations for about 30 PN.

Since 1983, however, the most important data in the IR are those collected by IRAS; up to now, about 950 IRAS sources have been identified as being PN. According to a sample of $100 \mathrm{PN}$, fluxes are given with the following qualities:

\begin{tabular}{lcccc} 
Quality & $12 \mu \mathrm{m}$ & $\mathbf{2 5} \mu \mathrm{m}$ & $60 \mu \mathrm{m}$ & $100 \mu \mathrm{m}$ \\
\cline { 2 - 5 } 1 (upper limit) & $55 \%$ & $9 \%$ & $15 \%$ & $79 \%$ \\
2 & $4 \%$ & $6 \%$ & $7 \%$ & $6 \%$ \\
3 & $41 \%$ & $85 \%$ & $78 \%$ & $15 \%$
\end{tabular}

Besides this, several hundred IRAS sources without optical counterparts presented IRAS colors typical of PN, and should be possible PN.

Table 4

\begin{tabular}{|c|c|c|c|c|}
\hline $\begin{array}{l}\text { Number of } \\
\text { objects }\end{array}$ & $\begin{array}{c}4.9 \mathrm{GHz} \\
(6 \mathrm{~cm})\end{array}$ & $\begin{array}{c}\text { Frequency } \\
14.7 \mathrm{GHz} \\
(2 \mathrm{~cm})\end{array}$ & Others & References \\
\hline 256 & & $\mathbf{x}$ & & Rubin R.H., 1970, Astron. Astrophys. 8, 171-180 \\
\hline 121 & & & $x$ & Higgs L.A., 1971, M.N.R.A.S. 153, 315-336 \\
\hline 74 & & & $\mathbf{x}$ & Aller L.H., Milne D.K., 1972, Austr. J. Phys. 25, 91-98 \\
\hline 165 & $\mathbf{x}$ & & & $\begin{array}{l}\text { Milne D.K., Aller L.H., 1975, Astron. Astrophys. } \\
\text { 38, 183-196 }\end{array}$ \\
\hline 167 & $x$ & & & Milne D.K., 1979, Astron. Astrophys. Suppl. 36, 227-235 \\
\hline 98 & & & $x$ & $\begin{array}{l}\text { Milne D.K., Webster B.L., 1979, Astron. Astrophys. } \\
\text { Suppl. 36, 169-171 }\end{array}$ \\
\hline 40 & $x$ & & $x$ & K wok S., 1981, Astrophys. J. 250, 232-239 \\
\hline 397 & & $\mathbf{x}$ & & $\begin{array}{l}\text { Milne D.K., Aller L.H., 1982, Astron. Astrophys. Suppl. } \\
\text { 50, 209-215 }\end{array}$ \\
\hline 42 & $x$ & $\mathrm{x}$ & $x$ & $\begin{array}{l}\text { Burton C.R., Feldman P.A., Marsh K.A., Allen D.A., } \\
\text { Wright A.E., 1982, M.N.R.A.S. 198, } 32 \text { i-338 }\end{array}$ \\
\hline 196 & & & $x$ & Calabretta M.R., 1982, M.N.R.A.S. 199, 141-150 \\
\hline 42 & $\mathbf{x}$ & & & $\begin{array}{l}\text { Gathier R., Pottasch S.R., Gross W.M., Gorkom J.H., } \\
\text { 1983, Astron. Astrophys. 120, 325-334 }\end{array}$ \\
\hline 62 & $\mathrm{x}$ & & $x$ & Isaacman R., 1984, M.N.R.A.S. 208, 399-408 \\
\hline 10 & $x$ & & $x$ & K wok S., 1985, Astron. J. 90, 49-58 \\
\hline$\approx 400$ & $x$ & & & $\begin{array}{l}\text { Zijlstra A., 1987, A VLA Survey of planetary nebulae, } \\
\text { Private communication }\end{array}$ \\
\hline
\end{tabular}




\section{- Radio fluxes}

The first important catalogue of radio data was done by Higgs (1971) for 557 PN. Accurate radio fluxes are known for about 450 objects (see Table 4). A VLA survey is being conducted by A.Zijlstra since 1986 and intends to cover the whole population of PN; the observations and reductions should be finished at the end of 1987; the results (total fluxes accurate to $5 \%$ ) will appear in the forthcoming Strasbourg-ESO catalogue of PN.

Low frequency $(<600 \mathrm{MHz}$ ) observations of $\mathrm{PN}$ have been reported in particular by Calabretta (1982, reference on Table 4, with references to previous works therein).

\section{Central stars}

A compilation of data for 480 nuclei of PN was published by Acker et al (1982), as finding charts for all these stars; "new" nuclei can now be seen on good quality ESO charts in particular (see the case of 298-4०1= NGC 4071). On the other hand, the true central star of very extended PN often remains unknown (as in the case of $27+16^{\circ} 1=\mathrm{DeHt}$ 2). One cannot say that the most probable candidate is the bluest star in the central region, because in several large $\mathrm{PN}$, the visible central star is a cold giant star (see the case of A 35, LoTr $5, \ldots)$.

A lot of spectroscopic observations have been done, by Mendez in particular. Kaler completed an "Atlas of PN central star spectra", containing data on 76 stars from 72 references.

5. The PN in Local Group Galaxies are catalogued by Jacoby and Ford and their collaborators. A catalogue of $315 \mathrm{PN}$ in $\mathrm{M} 31$ appeared in 1978 (ApJ Suppl.38), and was supplemented by 19 new PN in 1982 (ApJ 256) and by 37 $P N$ in 1987 (ApJ 317), bringing the total number of $P N$ catalogued in $M 31$ to 371. In addition, several papers giving finding charts and positions for $P N$ in M 32, NGC 147, NGC 185, and NGC 205 have been published. The same authors plan to publish a catalogue of the positions and magnitudes of $185 \mathrm{PN}$ in $M$ 81 and of 200 PN in NGC 5128.

\section{THE STRASBOURG - ESO CATALOGUE OF GALACTIC PLANETARY NEBULAE}

The new "Strasbourg - ESO Catalogue of Galactic Planetary Nebulae" which is in preparation since 1984 and which should appear in 1988, will be presented in book form; excerpts from the preliminary draft containing $102 \mathrm{PN}$ are available now, at Symposium 103 here in Mexico. The content of the forthcoming book is described in the "Announcement" published at the Vulcano Workshop (Acker, 1987). For those who wish, a computer version will be available, in the form of twelve specific "files", presenting the various data for PN ordered by their galactic PK number.

The data contained in the book (and in the files) are specified by bibliographical references collected at the "Centre de Donnees de Strasbourg" (CDS). From 1965 to mid-1987, the papers concerning individual PN or lists of PN represent 
a total of 3324 (excluding papers reporting only theoretical studies). As shown on figure 1, the annual number of publications is marked by the astronomical events devoted to $\mathrm{PN}$, and reflects the publication of the Proceedings of the IAU Symposiums on PN. Since 1970, thanks to the advent of "SIMBAD" at the CDS, it is possible to search the astronomical literature by object; this is how we have been able to see that the object with the largest number of bibliographical references in the whole Stellar Data Center which, on October 1st 1987, contained 688520 objects, of which 90000 are non-stellar objects, of which 70000 are galaxies, is NGC 7027 with 730 references from 1965 to now.

Another interesting fact is the large number of papers devoted to the determination of distances. Since the IAU Symposium 76 held at Ithaca in 1977, individual distances of PN have been given in 17 papers, and 9 statistical distance scales have been published; thus, the individual distance has been published for $111 \mathrm{PN}$, and one or several statistical values have been given for $679 \mathrm{PN}$ : the distance is known for more objects than is the exact radius (even though it is necessary for most distance scales) or the spectrum ! This does not prevent the distances from being extremely imprecise; improvements will only come from Hipparcos.

A good data catalogue will help towards the fruitful preparation of future science missions: Hipparcos (Ariane), HST (Shuttle) and ISO (Ariane), scheduled respectively in April 1989, mid 1989 and 1992-93.

A catalogue should set the record straight for current knowledge, but should also be a new departure point, for complementary observations and for research into new properties of PN.

\section{RE F E R E N C E S}

Acker A. : 1987, "Planetary and Proto-Planetary Nebulae : from IRAS to IS0", p. 35-38, A. Preite-Martinez (ed.), D. Reidel Publishing Company

Acker A., Gleizes F., Chopinet M., Marcout J., Ochsenbein F., Roques J.M. : 1982, "Catalogue of the central stars of true and possible planetary nebulae", Publication Spéciale du C.D.S. $N^{\circ} 3$ - Observatoire de Strasbourg, and Complements I (1982), II (1983), III (1984) - (480 stars)

Acker A., Marcout J., Ochsenbein F, Lortet M.C.: 1983, "Index and Cross-identification of PN ", Astron. Astrophys. Suppl. 54, 315-364

Fernandez A., Lortet M.C., Spite F. : 1983, Astron. Astrophys. Suppl. 52, $N^{\circ} 4$

Higgs L.A. : 1971, "Catalog of radio observations of PN and related optical data", P.A.B. Vol. $1, N^{\circ} 1$

Jaschek C. : 1984, Q. J. astr. Soc., 25, 259-266

Kaler J.B. : 1976, "Catalog of relative emission line intensities observed in PN and diffuse nebulae", Astrophys. J. Suppl. 31, 517-688

Perek L., Kohoutek L. : 1967, "Catalogue of galactic planetary nebulae", Praha, Academia Press, CSSR

Sabbadin F. : 1984, "Catalogue of expansion velocities of PN", Astron. Astrophys. Suppl. 58, 273-285

Schneider S.E., Terzian Y., Purgathofer A., Perinotto M. : 1983, "Catalog of radial velocities of PN", Astrophys. J. Suppl. 52, 399-423

Wray J.D. : 1966, "Study of Ha emission objects in the southern milky way", Thesis Ph.D. - Northwestern University 